\title{
$\beta$-Catenin Deficiency in Hepatocytes Aggravates Hepatocarcinogenesis Driven by Oncogenic $\beta$-Catenin and MET
}

\author{
Yan Liang ${ }^{1,{ }^{*}}$, Yun Feng ${ }^{1,2,{ }^{*}, \text { Min Zong }{ }^{1,{ }^{*}}, \text { Xufu Wei }^{1,3}, \text { Jin Lee }^{1} \text {, Yukuan Feng }}{ }^{4,5}$, Hairi Li $^{4}$, \\ Guangshun Yang $^{2}$, Zhong-Jun $\mathrm{Wu}^{3}$, Xiang-Dong $\mathrm{Fu}^{4}$, and Gen-Sheng Feng ${ }^{1, \#}$ \\ ${ }^{1}$ Department of Pathology, and Division of Biological Sciences, Moores UCSD Cancer Center, \\ University of California San Diego, La Jolla, California 92093, USA \\ ${ }^{2}$ The Fifth Department of Hepatic Surgery, Eastern Hepatobiliary Surgery Hospital, Second \\ Military Medical University, Shanghai 200438, P.R. China \\ ${ }^{3}$ Department of Hepatology, $1^{\text {st }}$ affiliated Hospital, Chong-Qing Medical University, China \\ ${ }^{4}$ Department of Cellular and Molecular Medicine, University of California San Diego, La Jolla, \\ California 92093, USA \\ ${ }^{5}$ Department of Anatomy, Mudanjiang Medical College, Mudanjiang, Heilongjiang 157011, China
}

\section{Abstract}

\begin{abstract}
Both activating and inactivating mutations in $c t n n b 1$, encoding $\beta$-catenin, have been implicated in liver tumorigenesis in humans and mice, although the underlying mechanisms are not fully understood. Herein we show that deletion of endogenous $\beta$-catenin in hepatocytes aggravated hepatocellular carcinoma (HCC) development driven by an oncogenic version of $\beta$-catenin (CAT) in combination with HGF receptor MET. Although the mitogenic signaling and cell cycle progression was modestly impaired after CAT/MET transfection, the $\beta$-catenin-deficient livers displayed changes in transcriptomes, increased DNA damage response, expanded Sox $9^{+}$cells, and upregulation of pro-tumorigenic cytokines including IL- 6 and TGF- $\beta 1$. These events eventually exacerbated CAT/MET-driven hepatocarcinogenesis in $\beta$-catenin-deficient livers, featured by upregulation of Erk, Akt and Wnt/ $\beta$-catenin signaling and cyclin D1 expression. The resultant mouse tumors showed similar transcriptomes to human HCC samples with concomitant CTNNB1 mutations and MET overexpression. These data argue that while dominantly activating mutants of $\beta$-catenin are oncogenic, inhibiting the oncogenic signaling pathway generates a pro-oncogenic microenvironment, which may facilitate $\mathrm{HCC}$ recurrence following a targeted therapy of the primary tumor. Therefore, an effective therapeutic strategy must require disruption of the oncogenic signaling in tumor cells and also suppression of the secondary tumor-promoting stromal effects in the liver microenvironment.
\end{abstract}

\footnotetext{
\# Corresponding to: Gen-Sheng Feng, gfeng@ ucsd.edu.

* Co-first authors
} 


\section{INTRODUCTION}

Primary liver cancer has been escalated to the second leading cause of cancer-related mortality worldwide, ${ }^{(1)}$ and hepatocellular carcinoma (HCC) accounts for approximately $90 \%$ of liver cancer cases. It is widely recognized that liver tumor initiation and progression are driven by both the altered intracellular signaling pathways and the hepatic microenvironment and, therefore, an effective therapeutic strategy requires disrupting both factors. So far, design of molecular targeted therapies has been mainly focused on blocking the identified tumor cell-intrinsic oncogenic signaling, which represents only one aspect of the pathogenic mechanisms. Therefore, it is not surprising that molecular targeted pharmaceuticals developed to date have achieved very limited therapeutic benefit for HCC patients. For example, Sorafenib, an FDA-approved drug for advanced HCC cases, can only extend patients' median survival for less than three months. ${ }^{(2)}$ Thus, development of more effective therapeutic strategies rely on elucidating how the hepatic microenvironment and the intracellular oncogenic signals synergistically drive liver tumorigenesis.

$\beta$-catenin is a central player in the Wnt signaling pathway, and mutations in CTNNB1, encoding $\beta$-catenin, have been detected in $20 \%-40 \%$ of HCC patients. ${ }^{(3,4)}$ Indeed, mutations or altered expression of $\beta$-catenin, MET and telomerase are the most frequently detected genetic lesions in large scale genome-wide screening of human HCC specimens. ${ }^{(5)}$ Consistently, transgenic mice expressing dominantly activating mutants of $\beta$-catenin exhibited increased susceptibility to HCC development. ${ }^{(6,7)}$ Paradoxically, ablating $\beta$ catenin in hepatocytes also resulted in elevated susceptibility to diethylnitrosamine (DEN)induced hepatocarcinogenesis in mice. ${ }^{(8,9)} \mathrm{A}$ most recent study demonstrated that the Wnt/ $\beta$-catenin signaling suppressed HCC formation by inhibiting a positive feedback loop between the Hippo and Notch pathways. ${ }^{(10)}$ Together, these studies revealed previously unrecognized complexity in the pathogenesis of liver cancer, which remains to be elucidated.

The present study was designed to interrogate a putative effect on hepatocarcinogenesis of the hepatic microenvironment induced by $\beta$-catenin loss in hepatocytes. Toward this goal, we co-transfected an oncogenic mutant of $\beta$-catenin $(\Delta \mathrm{N} 90-\beta$-catenin, CAT) and the human hepatocyte growth factor (HGF) receptor hMET into mice via hydrodynamic tail vein injection of plasmid DNA, and examined HCC development in wild-type (WT) and ctnnb $1^{\text {hep- }-}$ mouse livers. Interestingly, we found that $\beta$-catenin deficiency significantly exacerbated HCC driven by oncogenic $\beta$-catenin and hMET. The CAT/MET-induced tumors in $\beta$-catenin-deficient livers were characterized by more severe inflammation, increased activation of Wnt/ $\beta$-catenin, Erk and Akt signals, overexpression of cyclin D1 and expansion of Sox $9^{+}$cells. These results indicate that suppressing $\beta$-catenin in the liver induces a tumorpromoting stroma to aggravate liver cancer driven by mutant $\beta$-catenin and MET.

\section{MATERIALS AND METHODS}

\section{Mice and Treatment}

ctnnb1 $1^{\text {flox/flox }}$ mice obtained from the Jackson laboratory (B6.129-Ctnnb1tm2Kem/KnwJ) were crossed with Albumin-Cre (Alb-Cre) transgenic mouse (B6.Cg-Tg(Alb-cre)21Mgn/J), to generate hepatocyte-specific $\beta$-catenin knockout mice $\left(c t n n b 1^{\text {hep- }}--\right)$. The animal 
protocols (S09108) and all experimental procedures were approved by the UCSD Institutional Animal Care and Use Committee (IACUC). To establish the CAT/MET-induced HCC model, we performed hydrodynamic injection of plasmid DNAs into tail vein of 2month-old male mice following a previously published protocol. ${ }^{(11)}$ Each mouse received a total of $20.8 \mu \mathrm{g}$ plasmid DNAs, $10 \mu \mathrm{g}$ pT3-EF1a-c-MET(human); $10 \mu \mathrm{g}$ pT3-EF1a-AN90$\beta$-catenin (human) or $20 \mu \mathrm{g}$ pT3-EF1 $\alpha$ as vector control; and $0.8 \mu \mathrm{g} p C M V-S B$, encoding the sleeping beauty transposase and transposon. Mice were maintained on standard diet and sacrificed at indicated time points. The liver weight, body weight and liver tumor numbers and sizes were measured. A list of mice used in this study is provided in supporting table S5.

\section{Molecular and Cellular Analyses}

Immunoblotting, immunohistochemical (IHC) and immunofluorescence (IF) staining were performed as previously described. ${ }^{(12)}$ Cells with positive staining were scored in at least three fields. At least three mice were included in each group. Terminal deoxynucleotidyl transferase-mediated deoxyuridinetriphosphate nick end labeling (TUNEL) staining was performed as previously described. ${ }^{(12)}$ The apoptotic index was scored in at least three fields at $\times 200$ magnification/mouse. At least three mice were used in each group. Quantitative realtime polymerase chain reaction (qRT-PCR) was performed following standard protocol using Stratagene $m \times 3005 p$ machine. Detailed information for antibodies and qRT-PCR primer sequences are provided in supporting tables S6 and S7.

\section{RNA sequencing analysis}

Total RNAs were extracted from liver tissues using the RNeasy Microarray Tissue Mini Kit (Qiagen, cat. 73304) and RNA-sequencing (RNA-seq) was performed using the method multiplex analysis of polyA-linked sequences (MAPS), ${ }^{(13)}$ and the Illumina Hiseq2000 machine. All the RNA-seq raw data were aligned using STAR. ${ }^{(14)}$ Next, we performed gene differential expression analysis using Cuffdiff ${ }^{(15)}$ and R package Extraction of Differential Gene Expression (EDGE), ${ }^{(16,17)}$ respectively. Gene set enrichment analysis (GSEA) ${ }^{(18,19)}$ preranked tool and Ingenuity pathway analysis (IPA, Ingenuity ${ }^{\circledR}$ Systems, www.ingenuity.com) softwares were used for pathway analysis. To Compare the different expression levels of pathway related genes between KO and WT, we used the average logtransformed FPKM from pooled data in each group $(n=3)$. Kolmogorov-Smirnov test (KS test) was performed to determine the significance between two cumulative distributions. All the scatter, line and box plots were made with R package, ggplot2. ${ }^{(20)}$ All the human HCC RNA-seq data were collected from TCGA database LIHC project (https:// portal.gdc.cancer.gov/projects/TCGA-LIHC). A total of 371 cases were included in the analysis, in which 90 patients were found to have CTNNB1 mutations using cBioPortal. $(21,22)$ We then counted the mean and standard deviation (SD) of MET expression levels across the 90 samples represented by FPKM-UQ values and set the cutoff as mean $\pm \mathrm{SD}$. We identified 15 samples with concomitant MET overexpression and CTNNB1 mutations. Next, we made MDS plot between the selected 15 TCGA samples and our mouse data using function from $\mathrm{R}$ package edgeR. ${ }^{(23,24)}$ 


\section{Statistical Analysis}

Statistical analysis was conducted using GraphPad Prism 6 software. Data were presented as means $\pm \mathrm{SD}$, and statistical significance was calculated with a Student $t$ test, with $p<0.05$ considered significant.

\section{RESULTS}

\section{Ablating $\beta$-catenin in hepatocytes tempers liver development}

By breeding $c t n n b 1^{f l / f 1}$ and $A l b$-Cre mouse lines, we generated $c t n n b 1^{h e p-/}$ (KO) mice with $\beta$-catenin removed in hepatocytes (Supporting Fig. S1A). Immunoblot analysis demonstrated approximately $90 \%$ depletion of the protein in ctnnb $1^{\text {hep- }}$ - liver lysates compared to $W T$ controls, and detection of residual $\beta$-catenin in the mutants was apparently due to its expression in non-parenchymal cells (Supporting Fig. S1B). Removing $\beta$-catenin in hepatocytes mitigated the growth of liver mass, as indicated by modestly reduced ratios of liver versus body weights (LW/BW) measured at age of 2 months (Supporting Fig. S1C), consistent with previous reports. $(25,26)$ However, $\beta$-catenin removal did not cause significant changes in the histology or overall architecture of the liver (Supporting Fig. S1D). $\beta$-catenin was first identified as a binding partner of E-cadherin and cytoskeleton in mediating direct cell-cell adhesion. In the absence of $\beta$-catenin, $\gamma$-catenin may act to maintain adherens junctions (AJs), through association with E-cadherin and actin. We detected compensatory increase of $\gamma$-catenin expression in the livers of 8-week-old ctnnb $1^{\text {hep-/ }}$ mice (Supporting Fig. S2A), consistent with previous observations. ${ }^{(25,26)}$ While the expression of E-cadherin was modestly reduced in the mutant livers, no change was detected in $\mathrm{N}$-cadherin expression following $\beta$-catenin ablation (Supporting Fig. S2A).

\section{$\beta$-catenin deletion exacerbates HCC development driven by $\triangle \mathrm{N} 90-\beta$-catenin and MET}

To define a stromal effect of $\beta$-catenin deficiency in hepatocarcinogenesis, we transfected a constitutively active $\beta$-catenin ( $\Delta$ N90- $\beta$-catenin, CAT) and human MET (hMET) cDNAs into the liver through hydrodynamic tail vein injection together with a sleeping beauty transposase system. ${ }^{(27)}$ Using this approach, Chen's and other groups showed that delivery of the CAT/MET constructs rapidly induced HCC development in mice. ${ }^{(11,28)}$ Of note, simultaneous $C T N N B 1$ ( $\beta$-catenin) mutations and MET overexpression have been detected in $9 \%-12.5 \%$ human HCC samples. ${ }^{(7)}$

Immunostaining of liver sections demonstrated efficient delivery and expression of MET and CAT in the liver, using antibodies against hMET and the Myc-tag attached to $\Delta \mathrm{N} 90-\beta$ catenin mutant, as examined 3 days after plasmid injection (Fig. 1A). Many of the hMETpositive hepatocytes were also stained for the mutant $\beta$-catenin. PCR analysis of genomic DNAs and immunoblotting of liver lysates showed similar levels of CAT and MET expression in $W T$ and ctnnb1 $1^{\text {hep-/- }}$ mice (Fig. 1B, C). We performed immunostaining for $\mathrm{Ki} 67$ and hepatocyte marker HNF4a, and observed similar numbers of $\mathrm{Ki}^{+} 7^{+}$hepatocytes in $W T$ and ctnnb $1^{\text {hep-/ }}$ livers, examined 3 days after CAT/MET co-transfection (Fig. 1D). Meanwhile, TUNEL assay detected more apoptotic cells in the mutant than the WT livers (Fig. 1D). 
We then examined liver tumorigenesis after 7 weeks of CAT/MET injection, and observed much more and larger tumor nodules in $c$ tnnb $1^{\text {hep- }}{ }^{-}$than $W T$ livers (Fig. 1E). H\&E staining demonstrated less differentiated cells and more disordered structures in the mutant livers (Fig. 1E). The tumor numbers, maximal tumor sizes and liver versus body weight (LW/BW) ratios were much higher in $c$ tnnb $1^{\text {hep- } /}$ than the $W T$ mice (Fig. $1 \mathrm{~F}$ ). Immunostaining analysis showed that the tumors developed in both groups were positively stained for the mutant $\beta$-catenin (Fig. 1E). The number of Ki67-positive cells increased significantly in tumor areas in ctnnb $1^{\text {hep- }-}$ livers (Fig. 1D). Meanwhile, TUNEL staining of liver sections also revealed more apoptotic cells in the tumor areas, but not in surrounding normal tissues, of $c$ tnnb $1^{\text {hep }-1}$ livers, relative to the controls (Fig. 1D). Together, these results demonstrate more aggressive tumorigenesis driven by CAT/MET in $\beta$-catenin-deficient livers.

\section{$\beta$-catenin deficiency accelerates liver tumor cell expansion}

To determine if the heavier tumor burden in ctnnb $1^{\text {hep- } /-}$ mice is due to earlier tumor initiation or progression, we examined livers at 1 and 4 weeks after injection of CAT/MET plasmids or the empty vector. Up to 4 weeks after injection, no macroscopic tumors were observed in $W T$ or $c t n n b 1^{\text {hep- }-}$ livers (Fig. 2A). However, H\&E staining of liver sections detected microscopic tumor lesions with eosinophilic cytoplasm and disordered hepatocyte architecture in both groups injected with the oncogenes (Fig. 2A), while injection of the vector did not cause any significant pathological change in both groups (Supporting Fig. S2B). Compared to the $W T$, the $\beta$-catenin-deficient livers contained significantly more and larger tumor lesions, featured with enlarged and irregular nucleoli (Fig. 2A, C).

Immunohistochemical analysis detected similar numbers of mutant $\beta$-catenin ${ }^{+}$cells in $W T$ and ctnnb $1^{\text {hep- }-}$ livers, at 3 days and 1 week after plasmid injection, suggesting no effect of $\beta$-catenin deletion on the DNA transfection efficiency (Fig. 2B, C). However, we observed more mutant $\beta$-catenin ${ }^{+}$cells in $c$ tnnb $1^{\text {hep- }-}$ than $W T$ livers 4 weeks later (Fig. 2B, C). Consistently, Ki67 and HNF4a co-immunostaining showed significantly increased hepatocyte proliferation rate in $c t n n b 1^{\text {hep- } /}$ livers, compared to $W T$, at 4 weeks, with no difference at 1 week (Fig. 2D). Similar results were obtained for another cell proliferation marker PCNA by immunoblotting of liver lysates at week 4 (Supporting Fig. S7D). When examined at 7 weeks, all tumor nodules were derived from mutant $\beta$-catenin-positive cells (Fig. 2B). Taken together, these results suggest that $\beta$-catenin loss leads to accelerated tumor cell expansion driven by CAT/MET transfection.

\section{Transcriptomic analysis reveals disturbance of hepatic homeostasis by $\beta$-catenin removal}

The above data showed oddly that mutant $\beta$-catenin-driven HCC was exacerbated in $\beta$ catenin-deficient liver. To launch an unbiased and extensive query of underlying mechanisms, we performed RNA-seq analysis of transcriptomes in the livers at day 0 , day 3 or week 7. We built up a heatmap for these differentially expressed genes extracted using $\mathrm{R}$ package EDGE (Fig. 3A). Evidently, $\beta$-catenin loss had a profound impact on the hepatic transcriptomes, as examined at day 0 before delivery of the oncogenes, and also dramatically altered CAT/MET-induced gene expression patterns in the liver, examined 3 days or 7 weeks following the plasmid injection. The differentially expressed genes were clearly grouped into three clusters, each of which was up-regulated at a specific time point (Fig. 3A). 
Although the transcriptomic transition from healthy liver to tumor tissues could be distinguished similarly in the two genotypes, variations in the overall gene expression levels were remarkably enlarged in ctnnb $1^{\text {hep- }-}$ - livers compared to the $W T$, especially after oncogenes' transfection (Fig. 3A). Therefore, $\beta$-catenin removal severely disturbed the hepatic homeostasis, leading to changes in genome-wide gene expression in larger extents.

Next, we interrogated changes in specific pathways and upstream regulators based on differential gene expression analysis by Cuffdiff. With GSEA analysis, we found that ablating $\beta$-catenin in hepatocytes caused multiple changes in the hepatic microenvironment. Of note, dramatically upregulated in ctnnb $1^{\text {hep- }-}$ livers were pathways involved in innate immune and inflammatory responses, for example, interferons, tumor necrosis factor and interleukins (Fig. 3B, Supporting Fig. S3A, Supporting Table S1). Also changed significantly were secreted proteins in the extracellular matrix, with the small molecule metabolic processes down-regulated in $c t n n b 1^{\text {hep- } /}$ livers (Fig. 3B, Supporting Fig. S3A, Supporting Table S2).

\section{$\beta$-catenin loss attenuates CAT/MET-triggered mitogenic signaling and cell cycle gene expression in the liver}

We then examined up- or down-regulated genes acutely induced by CAT/MET transfection, by comparing the data at day 0 and day 3 (Supporting Fig. S4A). We identified 446 genes up-regulated in WTlivers, compared to 268 in ctnnb $1^{\text {hep- }-}$ livers, with only 103 commonly up-regulated in both groups. Conversely, more genes were down-regulated in the mutant than the WT livers (298 versus 201), having only 15 shared by the two groups. Comparative pathway analysis demonstrated that $\beta$-catenin removal had a robust inhibitory effect on CAT/MET-induced expression of E2f downstream target genes and cell cycle genes (Fig. 3C, Supporting Fig. S3B). We collected cell cycle-related genes from MSigDB (The Molecular Signatures Database $)^{(18,19)}$ and plotted their expression levels in the two groups at three time points. Similar to the pathway analysis, most of the genes changed significantly (shown as red dots) were down-regulated in mutant livers relative to the $W T$ at day 3 but not at day 0 or week 7 (Fig. 3D, Supporting Fig. S5A-B). To confirm the data, we selected 6 of these down-regulated cell cycle genes for further analysis. Consistently, qRT-PCR analysis demonstrated lower expression for five of them, Tbx3, Asns, Spc25, Cdkn1a and cyclin D1, in mutant than $W T$ livers at day 3 (Supporting Fig. S6A). Only 2 genes, Tbx3 and Asns, exhibited low expression in ctnnb $1^{\text {hep- }-}$ livers at day 0 , and 2 genes, Asns and cyclin D1, were up-regulated in mutants at week 7 . We further examined stage-specific cell cycle signature genes, ${ }^{(29)}$ and found that their expression levels were lower in mutant than $W T$ livers at day 3 (Supporting Fig. S5C). Using a similar approach, we also plotted HCC signature gene set from MSigDB (PATIL_LIVER_CANCER), and found surprisingly that the expression of these genes was also lower in ctnnb1 $1^{\text {hep- } /}$ than WT livers at day 3 (Fig. $3 \mathrm{E})$. Together, the RNA-seq analysis suggested that $\beta$-catenin deficiency diminished mitogenic signaling and cell cycle progression acutely triggered by CAT/MET transfection.

Cyclin D1 is known to release and activate E2f1 from its interaction with $\mathrm{Rb} 1,{ }^{(30)}$ and is a downstream target of Wnt/ $\beta$-catenin, Erk and Akt pathways. ${ }^{(28)}$ qRT-PCR and RNA-seq analysis detected lower expression of cyclin D1 in the mutant livers at day 3 (Supporting 
Fig. S6A-B). Immunoblotting detected similar levels of cyclin D1 in the two genotypes at day 0 , and modestly decreased amounts in ctnnb $1^{\text {hep- } /}$ livers, at day 3 , week 1 and 4 after CAT/MET transfection (Fig. 3F; Supporting Fig. S7C, D). The basal levels of p-Erk1/2, pJnk1/2, and p-P38 at day 0 were similar between control and ctnnb $1^{\text {hep- }-}$ livers (Fig. 3F; Supporting Fig. 7A). Exogenous CAT/MET expression did not have a significant impact on p-Erk, p-Jnk and p-p38 signaling in ctnnb $1^{\text {hep- }-}$ or $W T$ livers (Fig. 3F; Supporting Fig. S7A, C, D). Deleting $\beta$-catenin caused a modest increase of WNT7a, a key ligand for the Wnt/ $\beta$-catenin pathway, in 2-month-old livers, with no difference observed for WNT10a or c-Myc, as examined at day 0 and day 3 (Supporting Fig. S7A).

\section{Tumorigenesis is characterized by multiple pathway changes in ctnnb1 ${ }^{\text {hep-l- }}$ liver}

The overall impaired mitogenic signaling and cell cycle progression detected soon after CAT/MET transfection as described above would predict lower tumorigenesis in $c t n n b 1^{\text {hep- }}$ / - livers. We then focused the analysis on the tumor tissues isolated 7 weeks later, and found more pathways significantly changed in $\beta$-catenin-deficient livers (Fig. 4A). In particular, inflammation, hypoxia-related pathway and HIF1a transcription network were aberrantly elevated in tumors in $c$ tnnb $1^{\text {hep- } /-}$ livers. Using IPA to identify the upstream regulators, we found that the IL-6 pathway was activated more prominently in ctnnb $1^{\text {hep- }-}$ livers (Fig. 4B), and a set of genes known to be up-regulated by IL-6/Stat3 signaling were highly expressed in tumors of ctnnb1 $1^{\text {hep- }-}$ mice (Fig. 4C). Indeed, p-Stat3 levels were higher in tumor lysates of $c t n n b 1^{\text {hep- }-}$ than $W T$ livers (Fig. 4D), indicating upregulation of the IL-6/Stat3 signaling. It was reported most recently that IL- 6 trans-signaling promoted HCC development because of elevated DNA damages and $\beta$-catenin activation. ${ }^{(31)}$ We found that among the three genes significantly changed, comparing ctnnb $1^{\text {hep- } /}$ to WT, the expression of CXcl12 $(\log 2$ FoldChange $=-1.196, \mathrm{q}=0.00115)$ and $C d 74(-0.983, \mathrm{q}=0.00115)$ in the negative regulation dataset decreased, while the expression of a positively regulatory gene Rif1 $(1.594, \mathrm{q}=0.00882)$ increased in $c$ tnnb $1^{\text {hep- }-/}$ livers, indicating higher DNA damage response after $\beta$-catenin loss.

Immunoblotting detected higher levels of the exogenous $\Delta$ N90- $\beta$-catenin and hMET in tumor lysates of $c t n n b 1^{\text {hep- } /}$ mice (Fig. 4D), evidently due to drastic expansion of CAT/ MET-driven tumor cells in the mutant livers. p-Erk and p-Jnk signals were higher in ctnnb $1^{\text {hep- } /}$ liver tumors (Fig. 4D), with no difference in p-p38 (Supporting Fig. S7B), and the PI3K/Akt signaling increased modestly, as shown by p-Akt and p-FoxO1 signals (Fig. 4D). We also detected higher levels of c-Myc, p-c-Jun and c-Jun in ctnnb $1^{\text {hep- }-}$ than $W T$ liver tumors (Fig. 4D). The expression of cyclin D1 has been shown to associate with HCC development. ${ }^{(32)}$ cyclin D1 expression was lower in $c$ tnnb $1^{\text {hep- }}-1$ than $W T$ livers at early time points after plasmid injection as described above (Fig. 3F; Supporting Fig. S7C-D) but was remarkably elevated in ctnnb $1^{\text {hep- }-}$ - liver tumors at 7 weeks (Fig. 4D), consistent with qRT-PCR and RNA-seq data analysis (Supporting Fig. S6A-B). Immunostaining of liver sections showed more cyclin $\mathrm{D} 1^{+}$cells in $c$ tnnb $1^{\text {hep- } /}$ than $W T$ livers at week 7 (Supporting Fig. S6C), suggesting expansion of cyclin $\mathrm{D}^{+}$tumor cells in $\beta$-catenin-deficient liver. 


\section{Sox9-positive cell pool is expanded in ctnnb1 ${ }^{\text {hep- }- \text { - liver }}$}

Overexpression of the transcription factor Sox 9 (SRY-related high-mobility group box 9 ) has been found to confer stem cell properties in HCC through the canonical Wnt/ $\beta$-catenin pathway. ${ }^{(33,34)}$ Immunoblotting detected higher Sox9 expression in ctnnb $1^{\text {hep- } /}$ than $W T$ livers at day 0, and CAT/MET transfection induced further upregulation of Sox9, as examined 3 days later (Fig. 5A). Moreover, immunoblotting showed steadily elevated expression of Sox 9 in CAT/MET-driven tumor tissues in $c$ tnnb $1^{\text {hep- } /}$ livers at 7 weeks (Fig. 5A). qRT-PCR analysis demonstrated consistently higher mRNA levels of Sox 9 in $c t n n b 1^{\text {hep }}$ - - than WT livers, at day 0, 3 and week 7 after CAT/MET injection, suggesting upregulated Sox 9 gene transcription in $\beta$-catenin-deficient livers (Fig. 5B).

We then performed immunostaining to examine Sox 9 expression patterns and sources (Fig. 5C). At day 0 before the plasmid injection, Sox9-positive signals were mainly detected in the bile duct areas, and Sox $9^{+}$cells were more scattered in $c t n n b 1^{\text {hep- }}-1$ and $W T$ livers when examined at day 3. Further, we detected more Sox9-positive signals in the ctnnb $1^{\text {hep- }-}$ tumor areas at week 7 (Fig. 5C). Thus, Sox $9^{+}$cell expansion may be responsible, at least in part, for the enhanced tumorigenic phenotype in $\beta$-catenin-deficient livers. This may be in part due to signaling cross-talk between $\mathrm{Wnt} / \beta$-catenin and Erk pathway. ${ }^{(35)}$

\section{$\beta$-catenin loss induces a pro-tumorigenic stroma in the liver}

Next, we examined the hepatic microenvironment, and observed more inflammatory cells infiltrated in the ctnnb $1^{\text {hep- }-}$ livers, especially the $\mathrm{F} 4 / 80^{+}$macrophages, by immunostaining at day 3 after CAT/MET transfection (Fig. 6A). Staining for the monocyte marker Ly6G showed no difference between the two groups at day 3, but increased in ctnnb $1^{\text {hep- } /-}$ livers at week 7 . Macrophages tended to cluster in the tumor surrounding areas, with more $\mathrm{F} 4 / 80^{+}$ cells infiltrated into tumors in $c$ tnnb $1^{\text {hep- }-}$ livers (Fig. 6A). We did not observe dramatic difference in T, B lymphocytes between $W T$ and $c$ tnnb $1^{\text {hep- }}-1$ livers at week 7 (Fig. 6A). We also examined infiltration of inflammatory cells at week 1 and 4 after injection of CAT/MET plasmid or the vector (Supporting Fig. S8). By examining F4 $/ 80^{+}$macrophages and B220+ B cells, we found that the $\beta$-catenin-deficient livers exhibited more inflammatory responses to oncogene overexpression.

We examined local expression of inflammatory cytokines in the liver by qRT-PCR assay (Fig. 6B). Of note, the expression of $T G F-\beta 1$ and $I L-6$ was significantly higher in $c t n n b 1^{\text {hep }}$ - - than $W T$ livers in 2-month-old mice examined at day 0 . At 3 days after plasmid injection, elevated expression of $I L-12, T G F \beta 1, T N F a, I L-1 \beta$ and $I L-6$ was detected in the ctnnb $1^{\text {hep- }}$ - livers, compared to the $W T$. Higher expression of $I L-12, I L-1 \beta$ and $I L-6$ was persistently detected in the $c t n n b 1^{\text {hep- }-}$ than the $W T$ livers at 7 weeks after plasmid injection. Together, these results demonstrate that deleting $\beta$-catenin in hepatocytes generated a

microenvironment with increased expression of inflammatory cytokines that cooperate with oncogenic $\beta$-catenin and hMET signaling, resulting in aggravated HCC development.

\section{CAT/MET-induced tumor transcriptomes are shared in mice and humans}

The tumors isolated from $W T$ and $c t n n b 1^{\text {hep- } /}$ livers shared 382 up-regulated and 220 down-regulated genes, with only 19 genes changed oppositely between the two groups 
(Supporting Fig. 4B). Therefore, we reasoned that these 602 commonly up- or downregulated genes constituted a "tumor signature" induced by the CAT/MET oncogenic signals. We analyzed how the expression of these CAT/MET-induced signature genes were influenced by $\beta$-catenin loss, by examining their cumulative plots of fold changes at three time points. No significant difference was noted for the gene sets between $W T$ and $c t n n b 1^{\text {hep }}$ ${ }^{-1}$ livers at day 0 (Supporting Fig. S9). At day 3 after plasmid injection, the up-regulated group of genes decreased in the mutant livers (Fig. 7A), but increased in tumors of $c$ tnnb $1^{\text {hep }}$ -1- livers 7 weeks later (Fig. 7B). This result is consistent with the other data collectively showing impaired proliferative signaling in $c t n n b 1^{\text {hep- }-}$ liver at day 3 , but eventually more severe tumor loads in the mutants at week 7 .

By comparing the mouse tumor transcriptomes with human HCC samples in the databases, we detected significant correlation of the CAT/MET-driven tumors with a total of $371 \mathrm{HCC}$ samples, albeit with high degrees of variations as expected (Fig. 7C). However, in a comparison to a selective group of 15 human HCC samples with concomitant CTNNB1 mutations and MET overexpression (Supporting Table S3, S4), the correlation was much improved for the tumors in both $W T$ and $c t n n b 1^{\text {hep- } /-}$ mice (Fig. 7C), indicating high relevance of these mouse models with the human disease. Next, we made dimensionality reduction plots using functions from $\mathrm{R}$ package, edge $\mathrm{R},{ }^{(23,24)}$ by comparing differences between the 15 selected TCGA data with our data in higher dimensions (Fig. 7D). The dim1 was associated with batch effect, and $\operatorname{dim} 2$ represented progressive tumorigenesis, since our RNA-seq samples were ordered from day 0,3 to week 7 along dim2. This plotting showed that $c$ tnnb $1^{\text {hep- }-}$ - livers exhibited more severe tumorigenesis than the $W T$, and the 15 selected TCGA patient samples were positioned between day 3 and week 7 of the mouse data. Therefore, we believe that the CAT/MET-induced tumors in ctnnb $1^{\text {hep- }}{ }^{-}$livers is an ideal model to elucidate the synergy between the gained and lost functions of $\beta$-catenin in driving hepatocarcinogenesis.

\section{DISCUSSION}

Although it is widely recognized that the hepatic microenvironment plays a pivotal role in HCC pathogenesis, the underlying mechanisms are unclear. We have established a relatively simplified system to address this complicated issue, by injecting oncogenic $\beta$-catenin and MET into $\beta$-catenin-deficient and $W T$ livers. Interestingly, $\beta$-catenin deficiency dramatically aggravated CAT/MET-driven HCC development, which argues strongly that deleting the $W n t / \beta$-catenin pathway generates a tumor-promoting stroma in the liver. The tumors in $\beta$ catenin-deficient livers were hallmarked by increased inflammation, aberrant activation of Wnt $/ \beta$-catenin, Erk and Akt pathways, expansion of cyclin D1 ${ }^{+}$cells and Sox $9^{+}$cells, among other potential mechanisms.

Genome-wide screening of large cohorts of HCC patient samples detected co-activation of MET and Wnt/ $\beta$-catenin pathways, ${ }^{(7)}$ suggesting the requirement of their concerted effects in driving human HCC pathogenesis. Consistent to the human data, expressing a dominantly activating mutant of $c t n n b 1$ alone was insufficient to trigger liver tumorigenesis in mice, but simultaneous transfection of MET and $\beta$-catenin efficiently induced HCC, as reported by several groups. ${ }^{(7,28)}$ However, deleting $\beta$-catenin in hepatocytes also led to enhanced mouse 
susceptibility to HCC induced by DEN and phenobarbital. ${ }^{(9)}$ Similar to the phenotype of ctnnb $1^{\text {hep- }-}$ mice, we reported previously that deleting Shp2 also exacerbated DEN-induced HCC development. ${ }^{(36)}$ Nevertheless, the Shp $2^{\text {hep- } /}$ mice, despite displaying hepatic injuries, fibrosis and inflammation, were less susceptible to liver tumorigenesis driven by CAT/MET, unlike the ctnnb $1^{\text {hep- }-}$ mice (Liu et al., manuscript under revision). Thus, characterizing these two mouse HCC models clearly separated the cell-intrinsic oncogenic signaling from the microenvironmental effects in liver tumorigenesis.

As expected, deleting $\beta$-catenin in hepatocytes decreased liver weight and mitigated liver growth, consistent with a previous report, ${ }^{(25)}$ which is likely due to reduced basal level of hepatocyte proliferation and increased apoptosis. We also detected up-regulated expression of $\gamma$-catenin and normal adherens junctions in $c t n n b 1^{\text {hep- } /}$ livers. Interestingly, $\beta$-catenin haploinsufficiency induced aggressive tumor formation and metastasis through disruption of adherens junctions, but loss of both ctnnb1 alleles had only a minor impact on mammary tumorigenesis, due to the retention of normal adherens junctions. ${ }^{(37)}$

Following CAT/MET transfection, we detected increased hepatic infiltration of macrophages in ctnnb $1^{\text {hep- }-}$ mice, accompanied by elevated levels of inflammatory cytokines, such as IL-6, as revealed by RNA-seq and qRT-PCR analyses. A most recent report suggested involvement of the IL6-mediated inflammatory program in accelerating transformation of $\mathrm{CD}_{133^{+}}$liver stem cells into metastatic cancer stem cells. ${ }^{(38)}$ It was also reported that genomic instability arising during IL6-mediated liver regeneration in the context of chronic inflammation was linked to accelerated hepatocarcinogenesis. ${ }^{(39)}$ We believe that the elevated inflammatory responses, especially the IL-6 increase, account for, at least in part, the severe HCC development driven by CAT/MET transfection in the ctnnb $1^{\text {hep- } /}$ liver.

Several critical signaling pathways known to drive HCC, such as Wnt/ $\beta$-catenin, Erk, Stat3 and PI3K/Akt pathways, were over-activated in tumors of $c t n n b 1^{\text {hep- } /}$ livers, in association with expansion of cyclin $\mathrm{D}^{+}$and Sox $9^{+}$cells. The Erk/Akt-cyclin D1 route was previously shown to play a key role in the CAT/MET-induced HCC model. ${ }^{(28)}$ This study also demonstrated aberrant activation of the pathway in the liver tumors of $c$ tnnb $1^{\text {hep- } /}$ mice. Elevated expression of Sox 9 has been connected with more advanced tumor stages and poorer prognosis in HCC patients. ${ }^{(40)}$ Sox 9 was proposed as a novel liver CSC biomarker, (41) which confers the stemness property in HCC through Frizzled-7 mediated Wnt/ $\beta$-catenin signaling. ${ }^{(33)}$ A more recent report suggested a role of Sox 9 in promoting symmetrical cell division of CSCs in HCCs. ${ }^{(34)}$ We found that Sox 9 expression was remarkably higher in ctnnb1 $1^{\text {hep- } /}$ livers than controls, either before or after CAT/MET transfection, consistent with the severe tumorigenic phenotype.

It was interesting to note that the exogenous $\beta$-catenin-elicited mitogenic signaling and cell cycle progression were slightly impaired in $c$ tnn $b 1^{\text {hep- }-}$ livers, soon after plasmid injection. However, the RNA-seq analysis demonstrated that extracellular matrix and innate immune response-related pathways were significantly changed in ctnnb $1^{\text {hep- } /-}$ livers, even without oncogenes' transfection. More extensive changes, including immune responses, hypoxia and cell death, were observed in $c t n n b 1^{\text {hep- } /}$ liver tumors 7 weeks after expression of the oncogenes. Further, we detected markedly elevated expression and/or over-activation of 
transcription factors, such as Jun, E2f1 and HIF1a, etc. It is widely recognized that ECM is necessary to support the liver architecture, and also plays critical roles in modulating intracellular signal transduction and gene expression. ${ }^{(42)}$ Deregulation of ECM homeostasis may lead to cell transformation, tumorigenic angiogenesis and metastasis. Thus, the dramatic changes of ECM in ctnnb1 ${ }^{\text {hep- } /}$ livers, together with abnormally elevated inflammatory signals involving IL-6, may have contributed to the severe tumor phenotypes, by causing more infiltration of inflammatory cells, elevated DNA damages and liver injuries. Indeed, we detected much more cell death signals by TUNEL assay in the tumor areas than the non-tumor areas in ctnnb $1^{\text {hep- }-}$ livers.

In conclusion, data presented here illustrate a cooperative effect of the direct oncogenic signaling of mutant $\beta$-catenin and MET in hepatocytes with a hepatic tumor-promoting stroma induced by $\beta$-catenin deficiency (Fig. 7E), which argues for the complexity in targeting this most frequently activated pathway in HCC therapy. Disrupting the Wnt/ $\beta$ catenin pathway can block its direct oncogenic effect but meanwhile may induce a milieu of pro-tumorigenic stromal factors in the liver, thereby facilitating tumor relapse after treatment of primary HCC patients. A more effective therapeutic mean clearly requires simultaneous block to the primary oncogenic signals and the secondary tumor-enhancing factors arising in the hepatic microenvironment.

\section{Supplementary Material}

Refer to Web version on PubMed Central for supplementary material.

\section{Acknowledgments}

We wish to thank Drs. D Brenner, N Varki and our colleagues for helpful discussion, and for pathological examination of liver tumor samples, Dr. X Chen (UCSF) for generously providing the oncogene constructs. This project was supported by NIHR01CA176012 and R01CA188506 (to G.S.F.). Yan Liang was supported by a predoctoral fellowship of FISP.

\section{References}

1. Simard EP, Ward EM, Siegel R, Jemal A. Cancers with increasing incidence trends in the United States: 1999 through 2008. CA Cancer J Clin. 2012; 62:118-128. [PubMed: 22281605]

2. Llovet JM, Ricci S, Mazzaferro V, Hilgard P, Gane E, Blanc JF, de Oliveira AC, et al. Sorafenib in advanced hepatocellular carcinoma. N Engl J Med. 2008; 359:378-390. [PubMed: 18650514]

3. de La Coste A, Romagnolo B, Billuart P, Renard CA, Buendia MA, Soubrane O, Fabre M, et al. Somatic mutations of the beta-catenin gene are frequent in mouse and human hepatocellular carcinomas. Proc Natl Acad Sci USA. 1998; 95:8847-8851. [PubMed: 9671767]

4. Llovet JM, Zucman-Rossi J, Pikarsky E, Sangro B, Schwartz M, Sherman M, Gores G. Hepatocellular carcinoma. Nat Rev Dis Primers. 2016; 2:16018. [PubMed: 27158749]

5. Zucman-Rossi J, Villanueva A, Nault JC, Llovet JM. Genetic Landscape and Biomarkers of Hepatocellular Carcinoma. Gastroenterology. 2015; 149:1226-1239 e1224. [PubMed: 26099527]

6. Nejak-Bowen KN, Thompson MD, Singh S, Bowen WC Jr, Dar MJ, Khillan J, Dai C, et al. Accelerated liver regeneration and hepatocarcinogenesis in mice overexpressing serine- 45 mutant beta-catenin. Hepatology. 2010; 51:1603-1613. [PubMed: 20432254]

7. Tao J, Xu E, Zhao Y, Singh S, Li X, Couchy G, Chen X, et al. Modeling a human hepatocellular carcinoma subset in mice through coexpression of met and point-mutant beta-catenin. Hepatology. 2016; 64:1587-1605. [PubMed: 27097116] 
8. Zhang XF, Tan X, Zeng G, Misse A, Singh S, Kim Y, Klaunig JE, et al. Conditional beta-catenin loss in mice promotes chemical hepatocarcinogenesis: role of oxidative stress and platelet-derived growth factor receptor alpha/phosphoinositide 3-kinase signaling. Hepatology. 2010; 52:954-965. [PubMed: 20583210]

9. Rignall B, Braeuning A, Buchmann A, Schwarz M. Tumor formation in liver of conditional betacatenin-deficient mice exposed to a diethylnitrosamine/phenobarbital tumor promotion regimen. Carcinogenesis. 2011; 32:52-57. [PubMed: 21047994]

10. Kim W, Khan SK, Gvozdenovic-Jeremic J, Kim Y, Dahlman J, Kim H, Park O, et al. Hippo signaling interactions with $\mathrm{Wnt} /$ beta-catenin and Notch signaling repress liver tumorigenesis. J Clin Invest. 2017; 127:137-152. [PubMed: 27869648]

11. Tward AD, Jones KD, Yant S, Cheung ST, Fan ST, Chen X, Kay MA, et al. Distinct pathways of genomic progression to benign and malignant tumors of the liver. Proc Natl Acad Sci USA. 2007; 104:14771-14776. [PubMed: 17785413]

12. Zhu HH, Luo X, Zhang K, Cui J, Zhao H, Ji Z, Zhou Z, et al. Shp2 and Pten have antagonistic roles in myeloproliferation but cooperate to promote erythropoiesis in mammals. Proc Natl Acad Sci USA. 2015; 112:13342-13347. [PubMed: 26460004]

13. Fox-Walsh K, Davis-Turak J, Zhou Y, Li H, Fu XD. A multiplex RNA-seq strategy to profile poly(A+) RNA: application to analysis of transcription response and $3^{\prime}$ end formation. Genomics. 2011; 98:266-271. [PubMed: 21515359]

14. Dobin A, Davis CA, Schlesinger F, Drenkow J, Zaleski C, Jha S, Batut P, et al. STAR: ultrafast universal RNA-seq aligner. Bioinformatics. 2013; 29:15-21. [PubMed: 23104886]

15. Trapnell C, Hendrickson DG, Sauvageau M, Goff L, Rinn JL, Pachter L. Differential analysis of gene regulation at transcript resolution with RNA-seq. Nat Biotechnol. 2013; 31:46-53. [PubMed: 23222703]

16. Storey JD. A direct approach to false discovery rates. J Royal Stat Soc: Series B (Statistical Methodology). 2002; 64:479-498.

17. Storey JD, Tibshirani R. Statistical significance for genomewide studies. Proc Natl Acad Sci USA. 2003; 100:9440-9445. [PubMed: 12883005]

18. Mootha VK, Lindgren CM, Eriksson KF, Subramanian A, Sihag S, Lehar J, Puigserver P, et al. PGC-1alpha-responsive genes involved in oxidative phosphorylation are coordinately downregulated in human diabetes. Nat Genet. 2003; 34:267-273. [PubMed: 12808457]

19. Subramanian A, Tamayo P, Mootha VK, Mukherjee S, Ebert BL, Gillette MA, Paulovich A, et al. Gene set enrichment analysis: a knowledge-based approach for interpreting genome-wide expression profiles. Proc Natl Acad Sci USA. 2005; 102:15545-15550. [PubMed: 16199517]

20. Wickham H. ggplot2: Elegant Graphics for Data Analysis: Springer-Verlag New York. 2009

21. Cerami E, Gao J, Dogrusoz U, Gross BE, Sumer SO, Aksoy BA, Jacobsen A, et al. The cBio cancer genomics portal: an open platform for exploring multidimensional cancer genomics data. Cancer Discov. 2012; 2:401-404. [PubMed: 22588877]

22. Gao J, Aksoy BA, Dogrusoz U, Dresdner G, Gross B, Sumer SO, Sun Y, et al. Integrative analysis of complex cancer genomics and clinical profiles using the cBioPortal. Sci Signal. 2013; 6:11.

23. Robinson MD, McCarthy DJ, Smyth GK. edgeR: a Bioconductor package for differential expression analysis of digital gene expression data. Bioinformatics. 2010; 26:139-140. [PubMed: 19910308]

24. McCarthy DJ, Chen Y, Smyth GK. Differential expression analysis of multifactor RNA-Seq experiments with respect to biological variation. Nucleic Acids Res. 2012; 40:4288-4297. [PubMed: 22287627]

25. Tan X, Behari J, Cieply B, Michalopoulos GK, Monga SP. Conditional deletion of beta-catenin reveals its role in liver growth and regeneration. Gastroenterology. 2006; 131:1561-1572. [PubMed: 17101329]

26. Tan X, Apte U, Micsenyi A, Kotsagrelos E, Luo JH, Ranganathan S, Monga DK, et al. Epidermal growth factor receptor: a novel target of the Wnt/beta-catenin pathway in liver. Gastroenterology. 2005; 129:285-302. [PubMed: 16012954]

27. Chen X, Calvisi DF. Hydrodynamic transfection for generation of novel mouse models for liver cancer research. Am J Pathol. 2014; 184:912-923. [PubMed: 24480331] 
28. Shang N, Arteaga M, Zaidi A, Stauffer J, Cotler SJ, Zeleznik-Le NJ, Zhang J, et al. FAK is required for c-Met/beta-catenin-driven hepatocarcinogenesis. Hepatology. 2015; 61:214-226. [PubMed: 25163657]

29. Whitfield ML, Sherlock G, Saldanha AJ, Murray JI, Ball CA, Alexander KE, Matese JC, et al. Identification of genes periodically expressed in the human cell cycle and their expression in tumors. Mol Biol Cell. 2002; 13:1977-2000. [PubMed: 12058064]

30. Watanabe G, Albanese C, Lee RJ, Reutens A, Vairo G, Henglein B, Pestell RG. Inhibition of cyclin D1 kinase activity is associated with E2F-mediated inhibition of cyclin D1 promoter activity through E2F and Sp1. Mol Cell Biol. 1998; 18:3212-3222. [PubMed: 9584162]

31. Bergmann J, Muller M, Baumann N, Reichert M, Heneweer C, Bolik J, Lucke K, et al. IL-6 transsignaling is essential for the development of hepatocellular carcinoma. Hepatology. 2016

32. Musgrove EA, Caldon CE, Barraclough J, Stone A, Sutherland RL. Cyclin D as a therapeutic target in cancer. Nat Rev Cancer. 2011; 11:558-572. [PubMed: 21734724]

33. Leung CO, Mak WN, Kai AK, Chan KS, Lee TK, Ng IO, Lo RC. Sox 9 confers stemness properties in hepatocellular carcinoma through Frizzled-7 mediated Wnt/beta-catenin signaling. Oncotarget. 2016; 7:29371-29386. [PubMed: 27105493]

34. Liu C, Liu L, Chen X, Cheng J, Zhang H, Shen J, Shan J, et al. Sox9 regulates self-renewal and tumorigenicity by promoting symmetrical cell division of cancer stem cells in hepatocellular carcinoma. Hepatology. 2016; 64:117-129. [PubMed: 26910875]

35. Ling S, Chang X, Schultz L, Lee TK, Chaux A, Marchionni L, Netto GJ, et al. An EGFR-ERKSOX9 signaling cascade links urothelial development and regeneration to cancer. Cancer Res. 2011; 71:3812-3821. [PubMed: 21512138]

36. Bard-Chapeau EA, Li S, Ding J, Zhang SS, Zhu HH, Princen F, Fang DD, et al. Ptpn11/Shp2 acts as a tumor suppressor in hepatocellular carcinogenesis. Cancer Cell. 2011; 19:629-639. [PubMed: 21575863]

37. Tung B, Schade B, Cardiff RD, Aina OH, Sanguin-Gendreau V, Muller WJ. beta-Catenin haploinsufficiency promotes mammary tumorigenesis in an ErbB2-positive basal breast cancer model. Proc Natl Acad Sci USA. 2017; 114:E707-E716. [PubMed: 28096336]

38. Mitra A, Yan J, Xia X, Zhou S, Chen J, Mishra L, Li S. IL6-mediated inflammatory loop reprograms normal to epithelial-mesenchymal transition+ metastatic cancer stem cells in preneoplastic liver of transforming growth factor beta-deficient beta2-spectrin+/- mice. Hepatology. 2017; 65:1222-1236. [PubMed: 27863449]

39. Lanton T, Shriki A, Nechemia-Arbely Y, Abramovitch R, Levkovitch O, Adar R, Rosenberg N, et al. Interleukin 6-dependent genomic instability heralds accelerated carcinogenesis following liver regeneration on a background of chronic hepatitis. Hepatology. 2017; 65:1600-1611. [PubMed: 28027584]

40. Guo X, Xiong L, Sun T, Peng R, Zou L, Zhu H, Zhang J, et al. Expression features of SOX9 associate with tumor progression and poor prognosis of hepatocellular carcinoma. Diagn Pathol. 2012; 7:44. [PubMed: 22515642]

41. Kawai T, Yasuchika K, Ishii T, Miyauchi Y, Kojima H, Yamaoka R, Katayama H, et al. SOX9 is a novel cancer stem cell marker surrogated by osteopontin in human hepatocellular carcinoma. Sci Rep. 2016; 6:30489. [PubMed: 27457505]

42. Hernandez-Gea V, Toffanin S, Friedman SL, Llovet JM. Role of the microenvironment in the pathogenesis and treatment of hepatocellular carcinoma. Gastroenterology. 2013; 144:512-527. [PubMed: 23313965] 

ctnnb1 ${ }^{w t / w t}: A l b-C r e^{+}$. transgenes in liver tissues 3 days after plasmid injection.
A
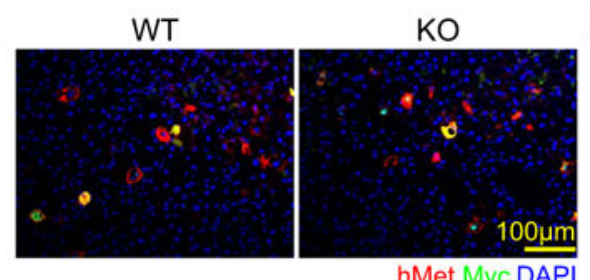

B

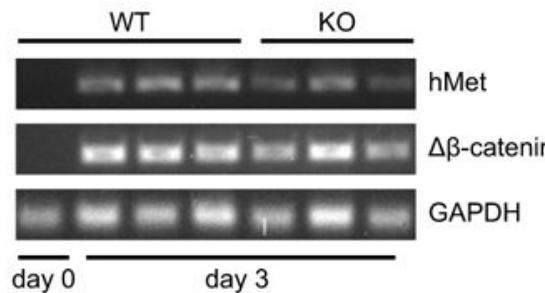

E
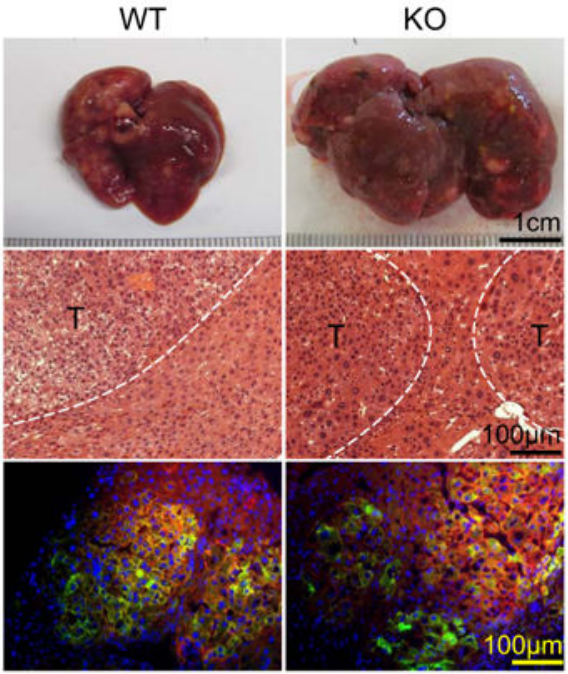

hMet Myc DAPI

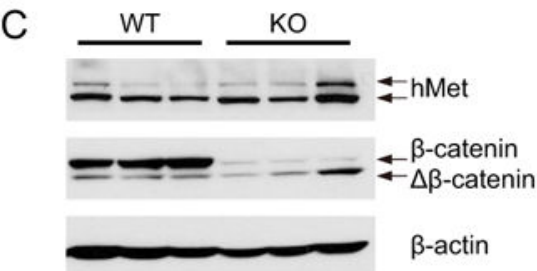

D

WT

$\mathrm{KO}$
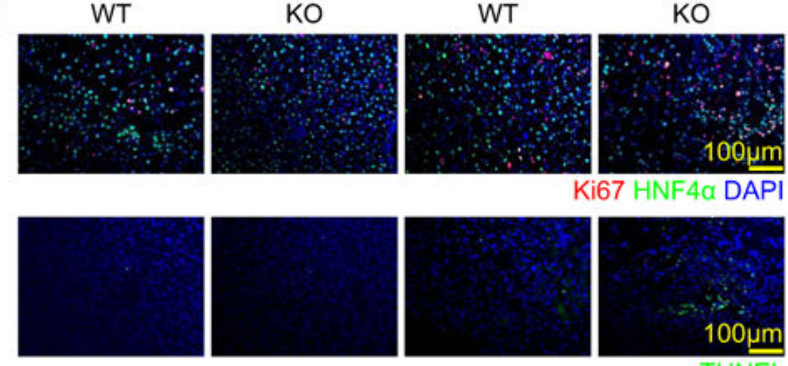

day 3
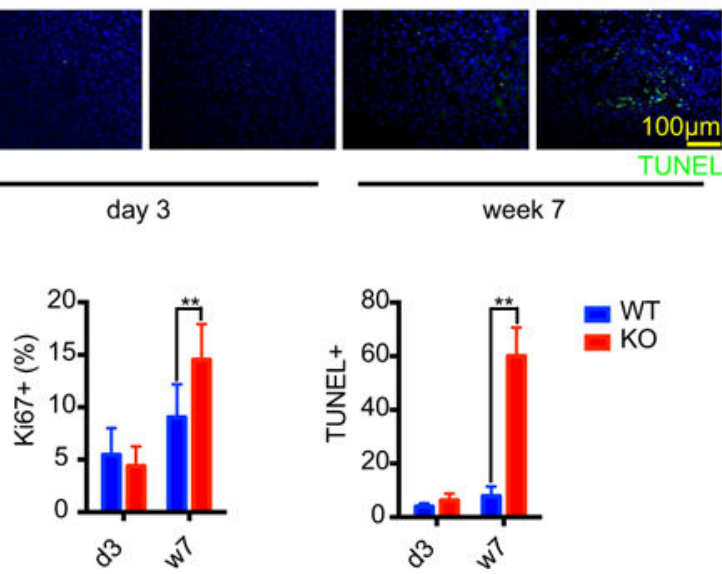

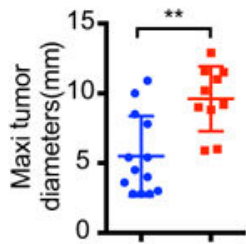

$\mathrm{F}$
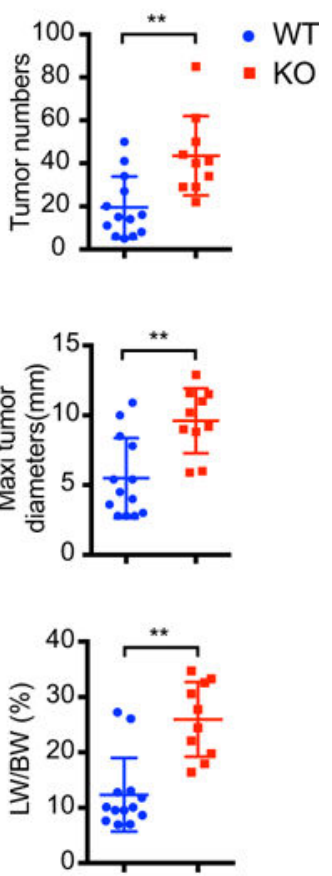

Figure 1. $\beta$-catenin deletion exacerbates HCC development driven by $\triangle \mathrm{N} 90$ - $\beta$-catenin and MET (A) Representative pictures of co-staining for hMet and $\Delta \mathrm{N} 90-\beta$-catenin (Myc-tag) in wildtype (WT) and $c t n n b 1^{\text {thep }}(\mathrm{KO})$ mice 3 days after CAT/MET injection. Control mice (WT) used in this study included ctnnb $1^{\text {flox/flox }: A l b-C r e}{ }^{-}, c_{n n n} 1^{f l o x} / w t: A l b-C r e^{-}$and

(B) PCR analysis of genomic DNA for exogenous hMet and $\Delta N 90-\beta$-catenin ( $\Delta-\beta$-catenin) 
(C) Immunoblot analysis of hMet, $\Delta$ - $\beta$-catenin and endogenous $\beta$-catenin in liver lysates 3 days after injection of plasmids.

(D) Top: representative Ki67 immunostaining and TUNEL assay of liver sections 3 days or 7 weeks after CAT/MET injection. Bottom: quantification of $\mathrm{Ki}^{+} 7^{+}$or TUNEL ${ }^{+}$cells $(\mathrm{n}=3)$. * $\mathrm{p}<0.05 . * * \mathrm{p}<0.01$.

(E) Top: macroscopic view of representative livers 7 weeks after hydrodynamic injection. Middle: representative H\&E staining of liver sections. Bottom: representative coimmunostaining of exogenous hMet and $\Delta \mathrm{N} 90-\beta$-catenin (Myc) on liver sections. T: tumor area.

(F) Tumor numbers, maximal tumor diameters and liver weight/ body weight (LB/WB) ratios were compared $(n=10-13)$. Data are expressed as means $\pm S D, * * p<0.01$. 
A

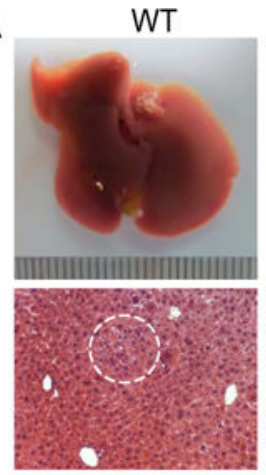

C
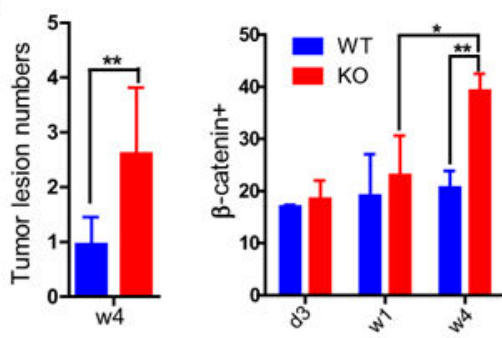

D
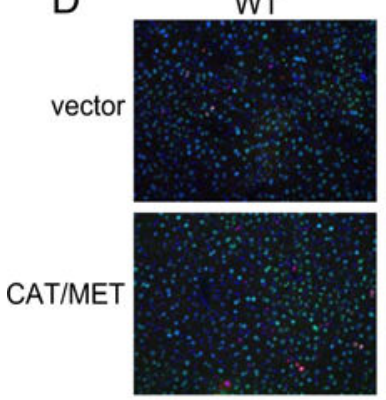

week1

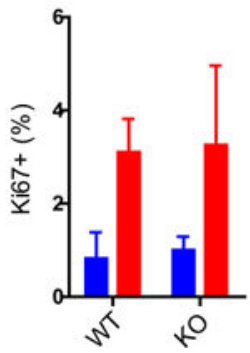

$1 \mathrm{~cm}$

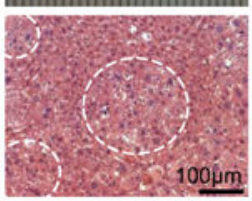

B

WT

day 3

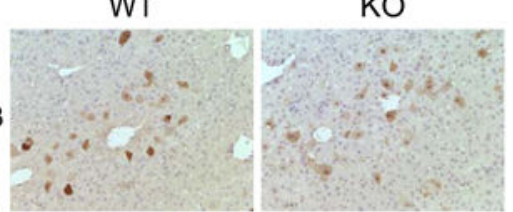

week 1
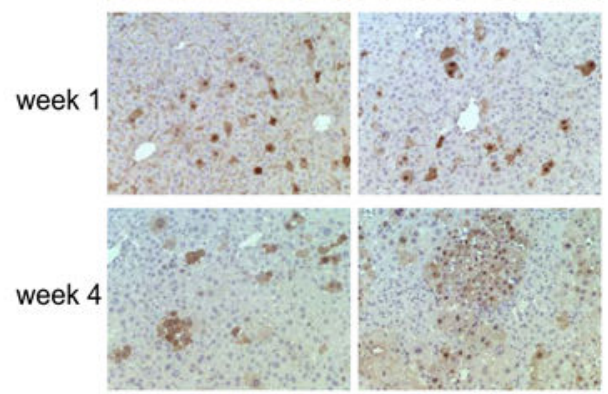

week 7
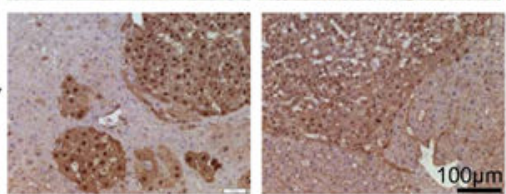

E
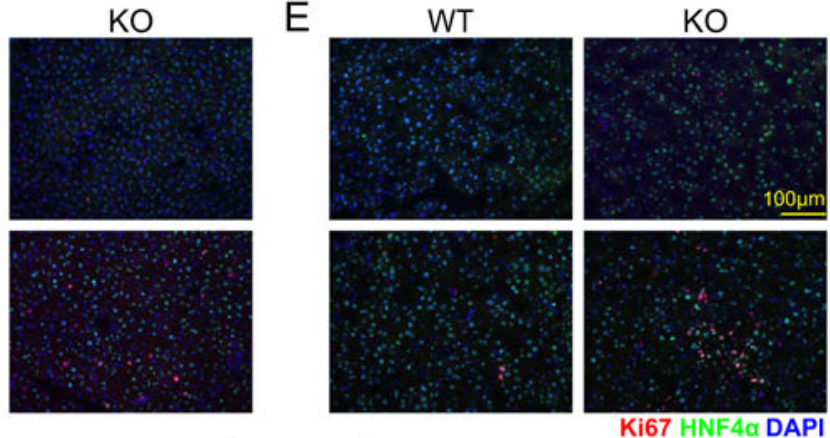

week 4

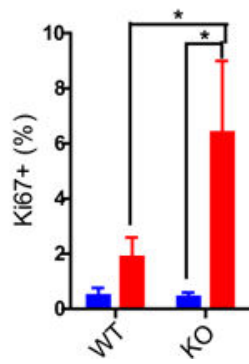

vector

CAT/MET

Figure 2. $\beta$-catenin deficiency accelerates liver tumor cell expansion

(A) Macroscopic view of representative livers (Upper panels), and H\&E-stained sections (lower panel, white circles indicate tumor nodules) at week 4 after plasmid injection.

(B) $\Delta \beta$-catenin-positive cells were examined by immunostaining with anti-Myc antibody in $W T$ and $K O$ livers at indicated time points after injection of plasmids. Tumor nodules were derived from $\Delta \beta$-catenin-positive cells as examined at week 7 .

(C) The numbers of tumor lesion were quantified at week 4 , and the $\Delta \beta$-catenin-positive cells were counted at day 3 , week 1 and $4, \mathrm{n}=3-4, * \mathrm{p}<0.05, * * \mathrm{p}<0.01$. 
(D-E) Hepatocyte proliferation was examined by Ki67 immunostaining of liver sections injected with vector control and CAT/MET at week 1 (D) or week 4 (E). $n=3-4,{ }^{*} \mathrm{p}<0.05$. 
A

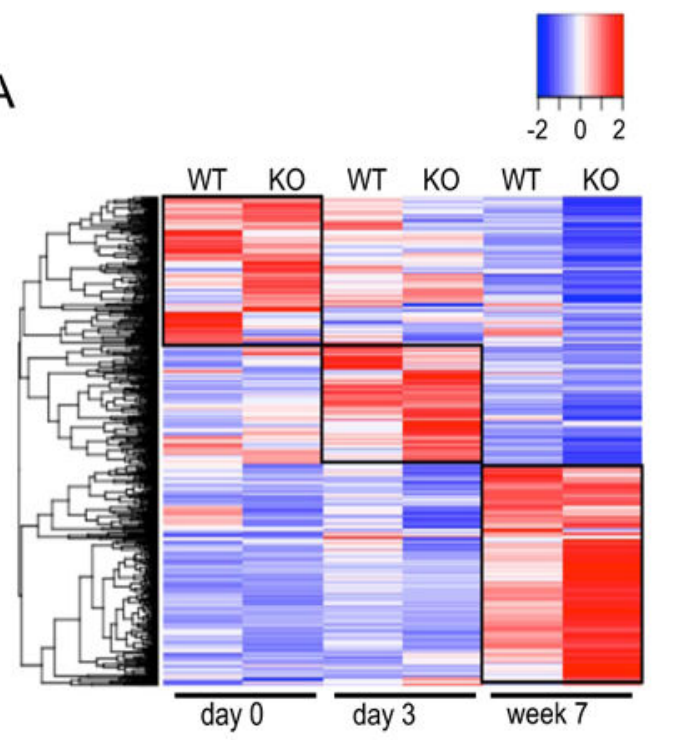

C

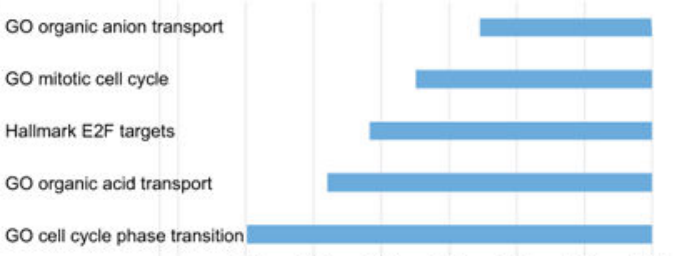

$\begin{array}{lllllll}-2.7 & -2.6 & -2.5 & -2.4 & -2.3 & -2.2 & -2.1\end{array}$

$E$

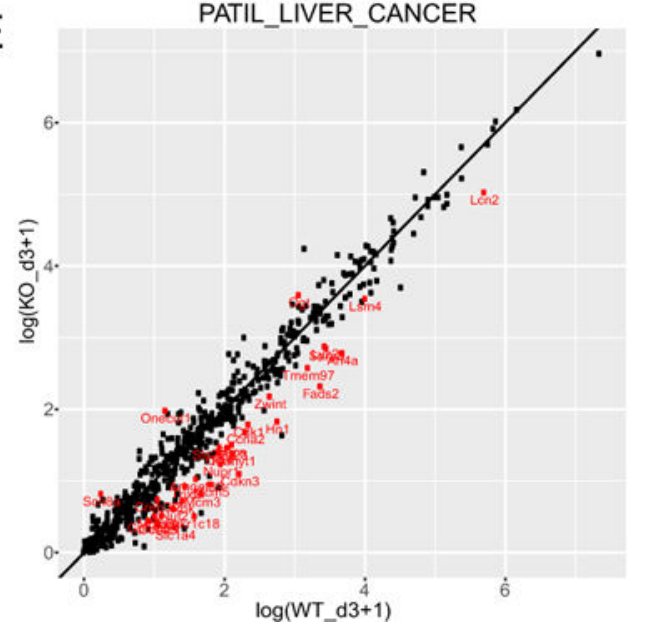

B

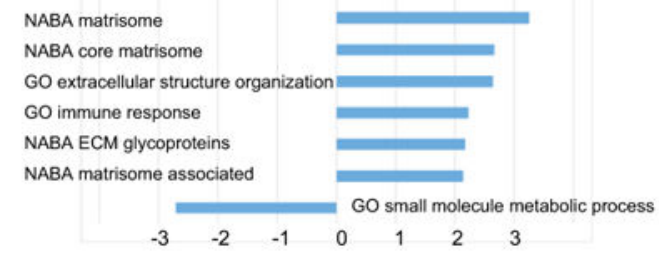

D

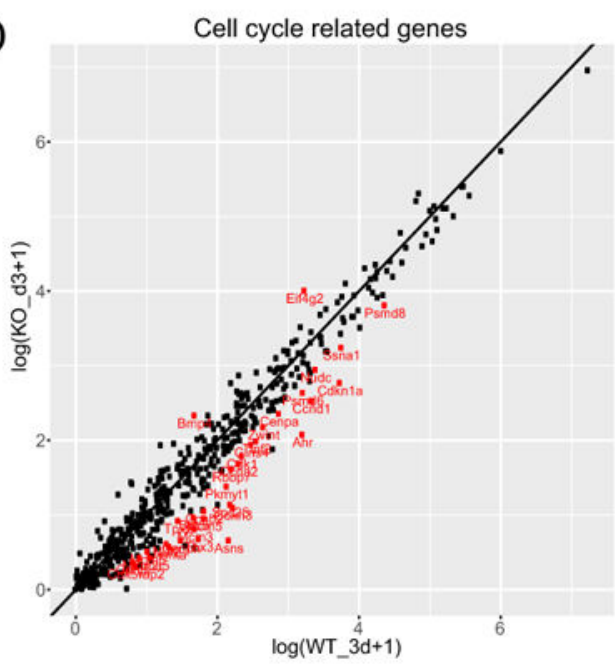

$\mathrm{F}$

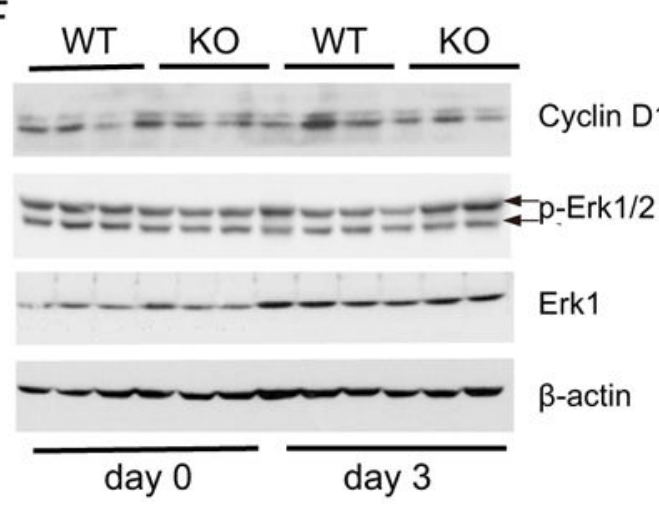

Figure 3. $\beta$-catenin loss disturbs overall hepatic gene expression profiles

(A) A heatmap built with RNA-seq data shows significantly changed gene expression patterns genome-wide. Each row represents mean values of 3 replicated WT and ctnnb1 ${ }^{\text {hep-/ }}$ - $(\mathrm{KO})$ liver samples in one group $(\mathrm{n}=3)$. Color scale is provided as references.

(B) Positive or negative pathways (up- or down-regulated in KO livers, relative to WT) at age of 8 weeks were identified by GSEA. The abscissa is NES value.

(C) Negative pathways (down-regulated in KO livers 3 days after CAT/MET injection) were found by GSEA. The abscissa is NES value. 
(D) Comparison of log-transformed expression levels of cell cycle-related genes between WT and KO livers at 3 days after injection. These cell cycle-related genes were collected from 4 separate gene sets in Msigdb, including KEGG_CELL_CYCLE, MITOTIC_CELL_CYCLE, CELL_CYCLE_GO_0007049 and

REACTOME_CELL_CYCLE. Significantly changed genes were shown in red dots. (E) Comparison of log-transformed expression levels of liver cancer-related genes between WT and KO livers at 3 days after injection. These liver cancer-related genes were from gene set PATIL_LIVER_CANCER in Msigdb. Genes changed significantly were shown in red dots.

(F) Immunoblot analysis of Cyclin D1, p-Erk1/2, and Erk1 at day 0 or 3 of WT and KO livers. 
A

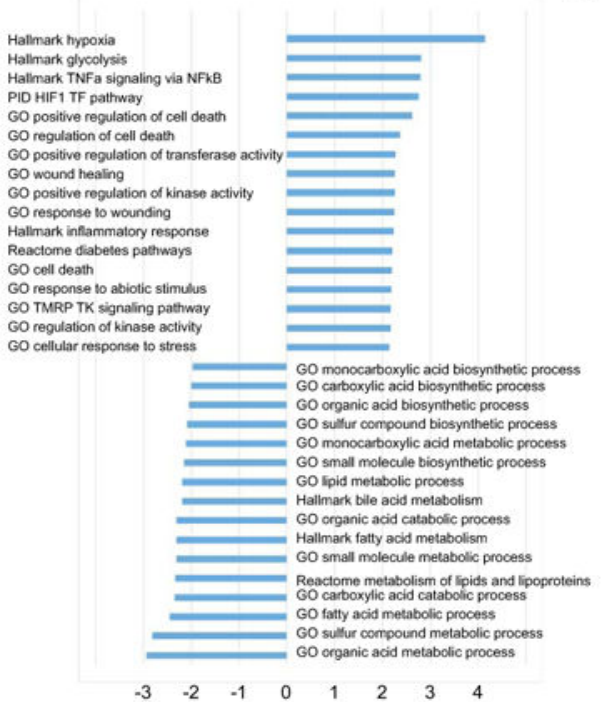

B
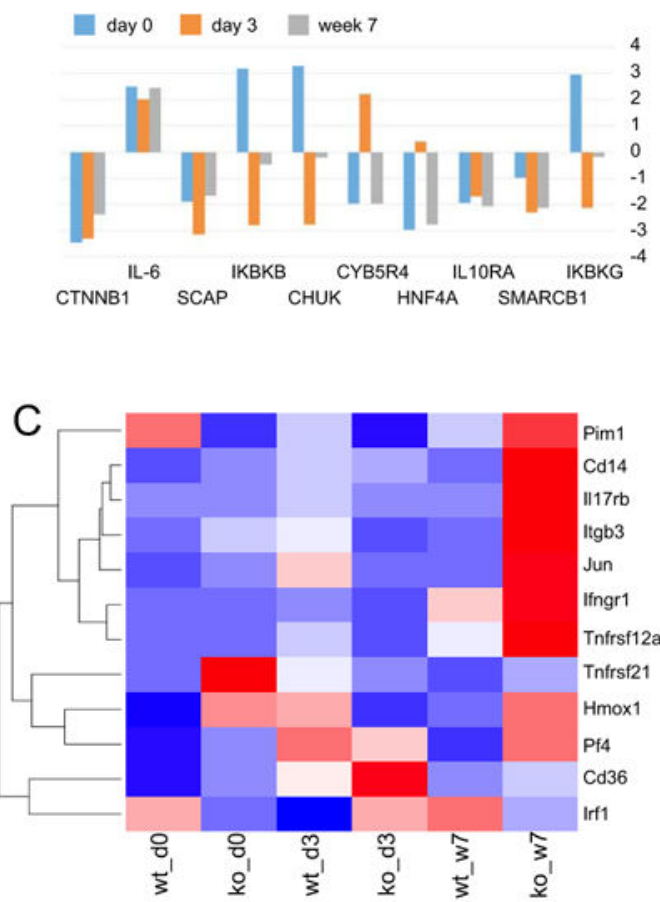
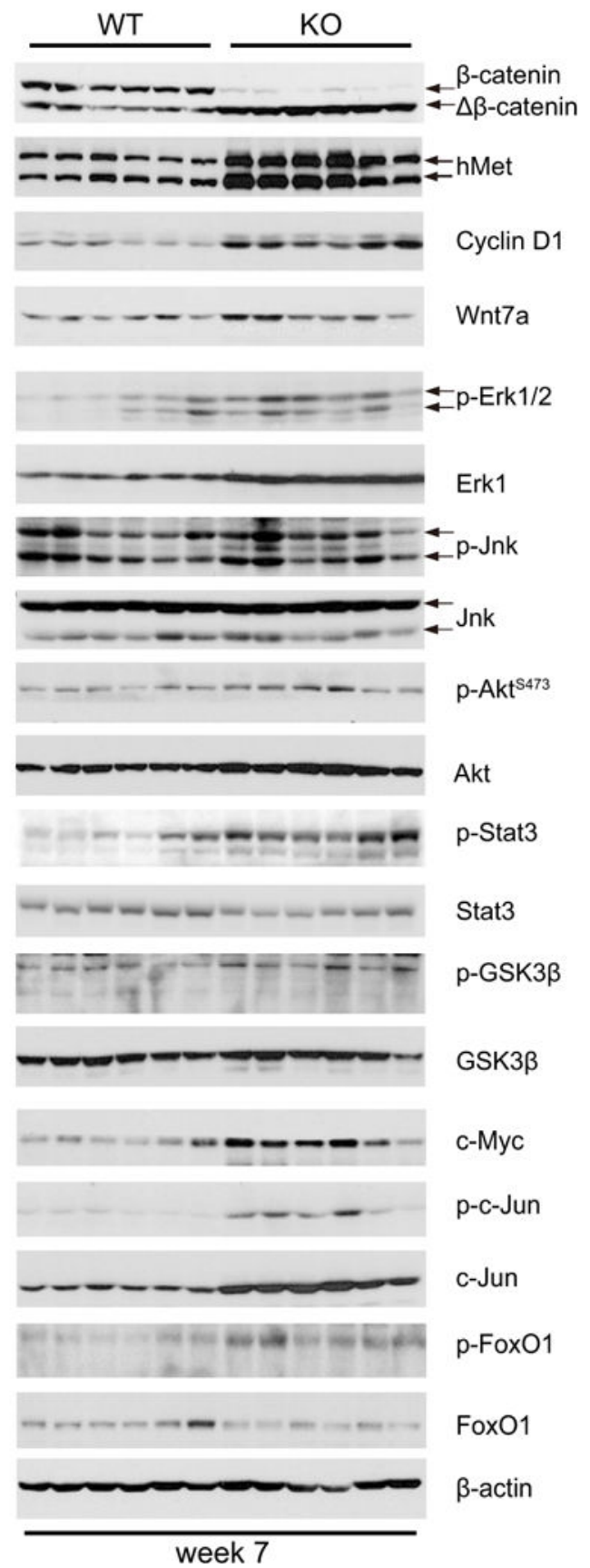

\section{K3 $\beta$}

-Myc

-C-Jun

-Jun

FoxO1

xO1

-actin

Figure 4. Tumorigenesis is characterized by multiple pathway changes in ctnnb1 $^{\text {hep-l- }}$ liver (A) Positive and negative pathways in tumors isolated at 7 weeks after CAT/MET injection were identified by GSEA as up- and down-regulated in $c t n n b 1^{\text {hep- }-/}$ livers, compared to WT control. The abscissa is NES value.

(B) Activation and inhibition of upstream regulators were analyzed by IPA in $c t n n b 1^{\text {hep- } /}$ to $W T$ livers at three time points. Data are represented by z-score. Z-score $>2$ indicates activation, and z-score $<-2$ indicates inhibition.

Hepatology. Author manuscript; available in PMC 2019 May 01. 
(C) Heatmap of genes related to the IL-6/Stat3 pathway with significant changes comparing WT and ctnnb1 $1^{\text {hep- }}-$ (KO) livers at day 0,3 or week 7.

(D) Immunoblotting analysis of signaling pathways in liver tumor lysates derived from $W T$ and $\mathrm{KO}$ mice. 

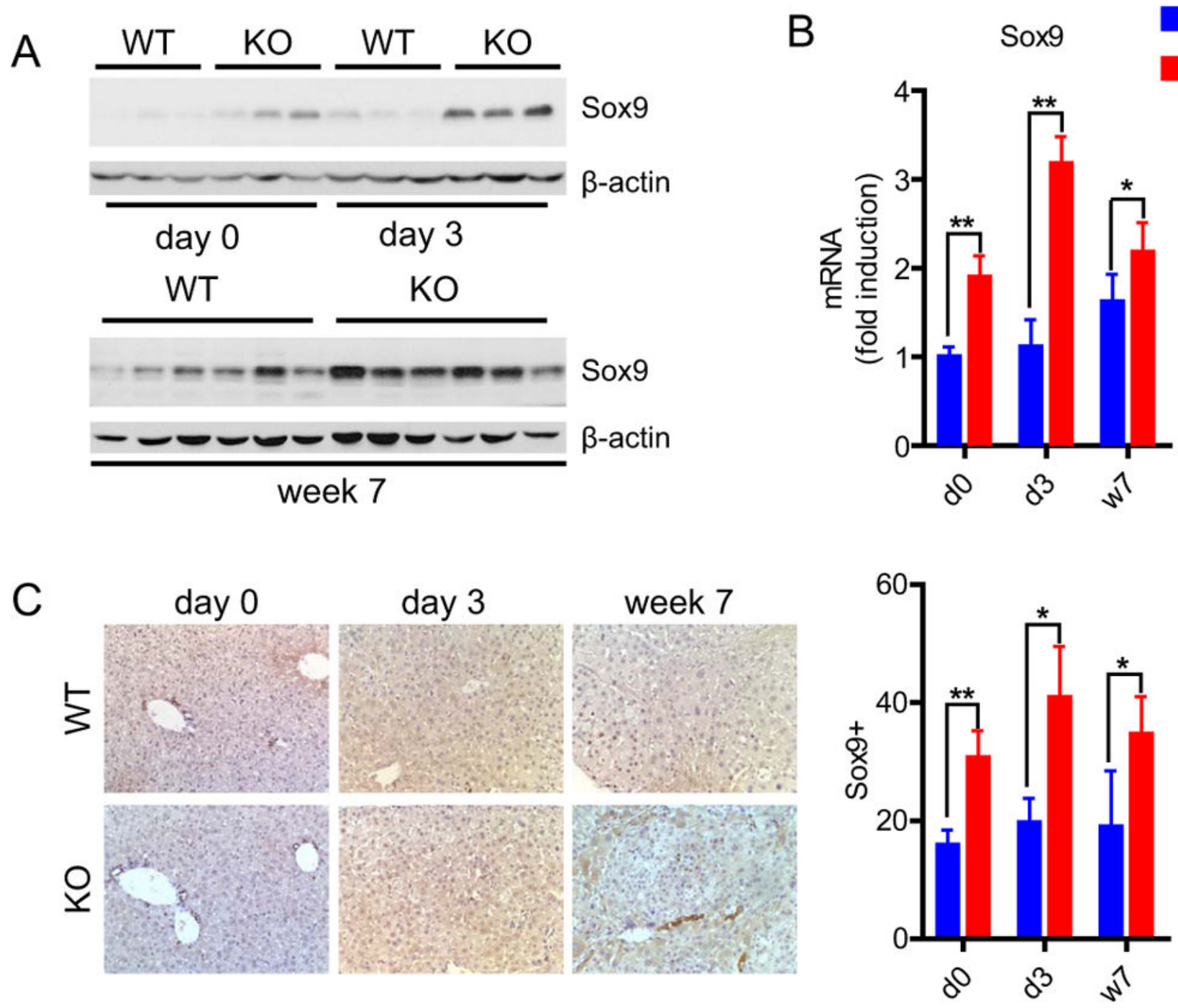

Figure 5. Sox9-positive cell pool is expanded in ctnnb $^{\text {hep-/- }}$ liver

(A) Protein levels of Sox9 at different time points were examined by immunoblotting of liver lysates.

(B) mRNA levels of Sox9 at different time points were measured by real-time quantitative PCR $(n=3)$.

(C) Representative Sox9 immunostaining of liver sections from $W T$ and $c t n n b 1^{\text {hep- }-}$ (KO) mice and quantification of Sox9-positive cells (x 200) on liver sections ( $\mathrm{n}=3$ ). Statistic data are shown as means \pm SD. $* \mathrm{p}<0.05 . * * \mathrm{p}<0.01$. 

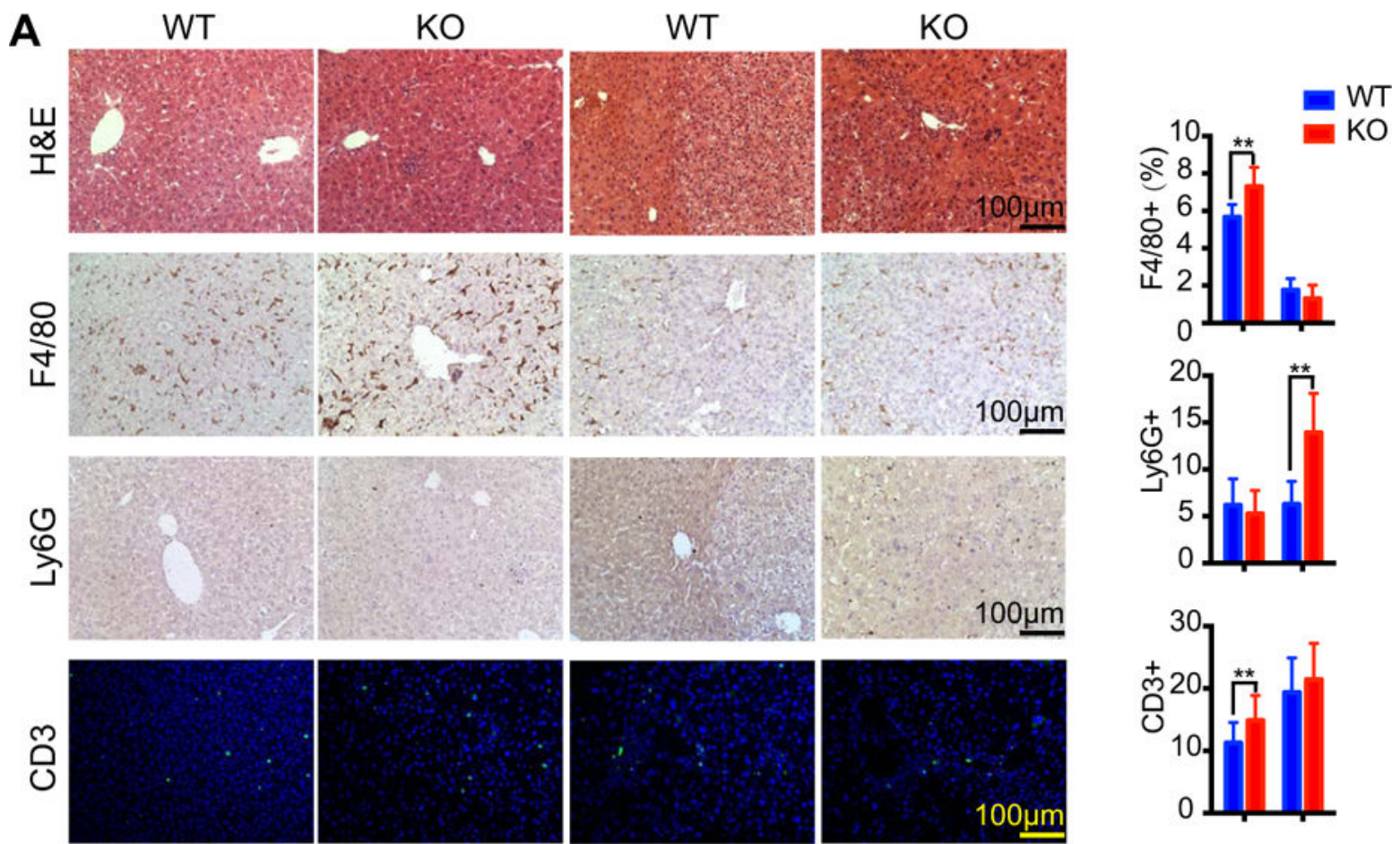

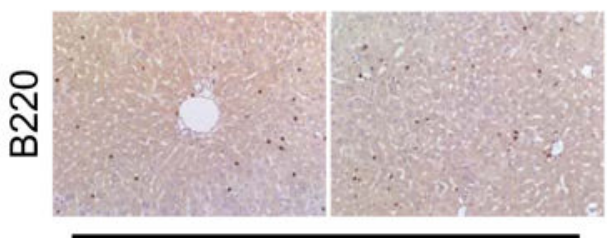

day 3
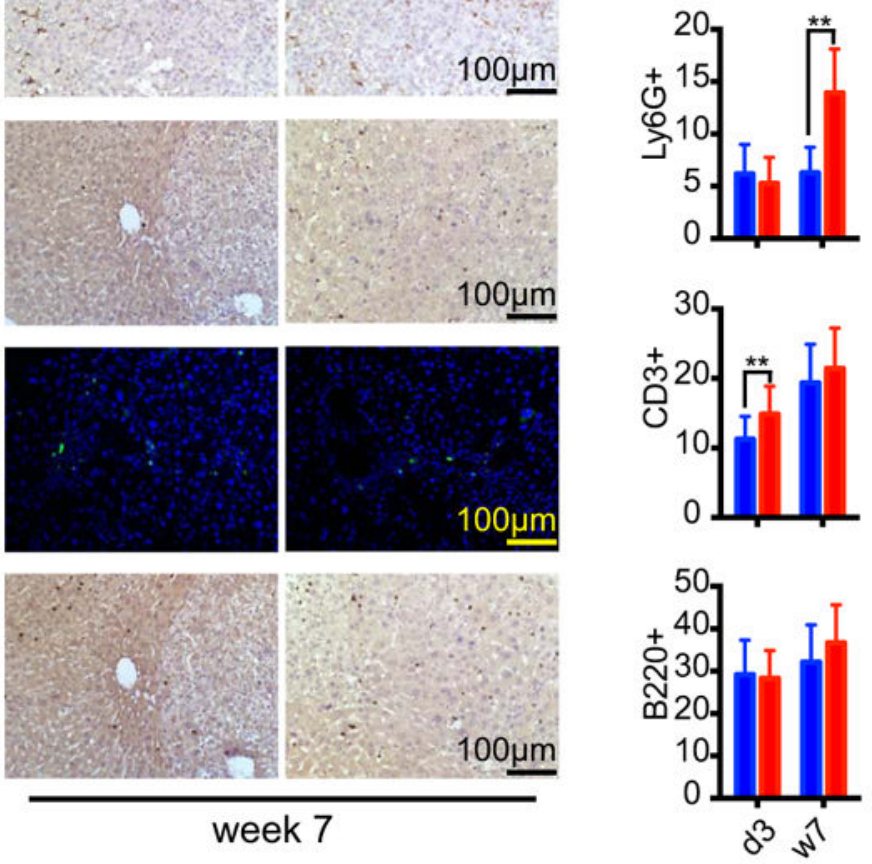

B
IL-12

TGF- $\beta 1$

TNF- $\alpha$

$\mathrm{IL}-1 \beta$

IL-6
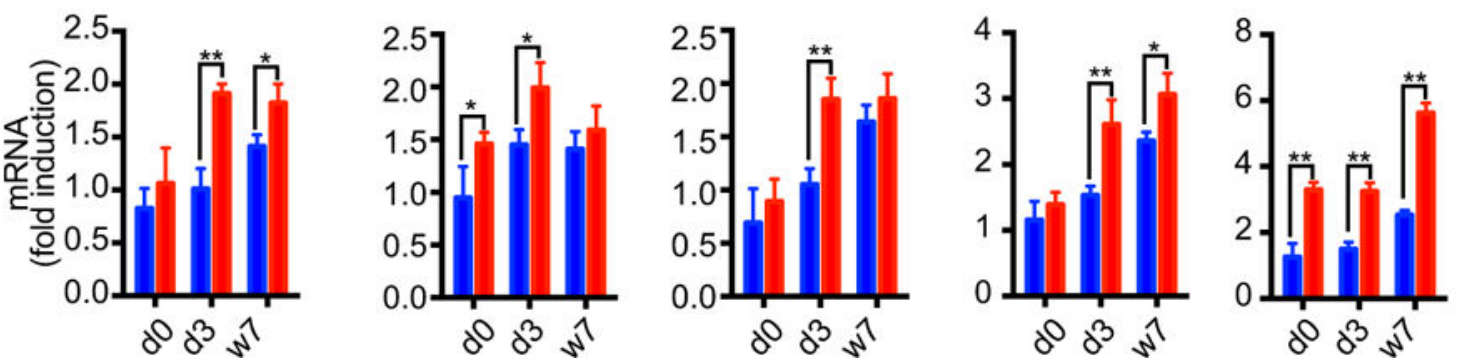

Figure 6. $\beta$-catenin loss induces a pro-tumorigenic stroma in the liver

(A) Left: representative $\mathrm{H} \& \mathrm{E}$ and immunostaining of immune cells in the livers. Right: quantification of Ly6G, CD3, and B220-positive cell numbers and percentage of positive $\mathrm{F} 4 / 80$ staining areas $(\mathrm{n}=3)$. $* * \mathrm{p}<0.01$.

(B) mRNA levels of inflammatory cytokine genes were determined by real-time quantitative PCR, $\mathrm{n}=3 ; * \mathrm{p}<0.05 ; * * \mathrm{p}<0.01$. 
A

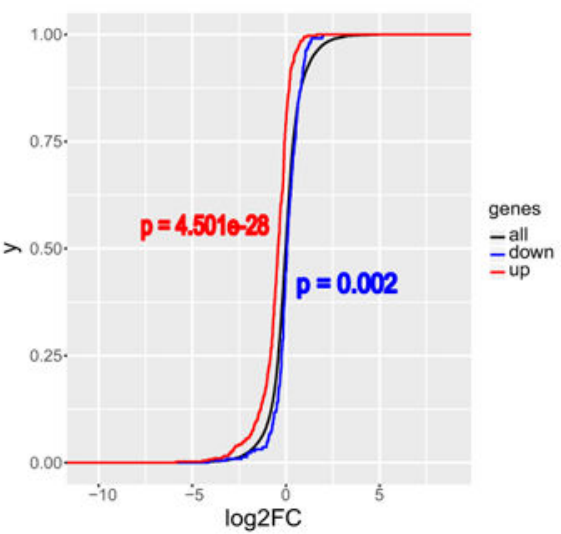

C

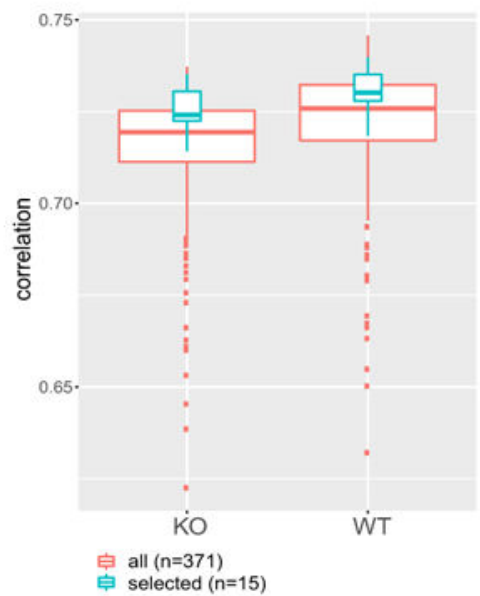

B

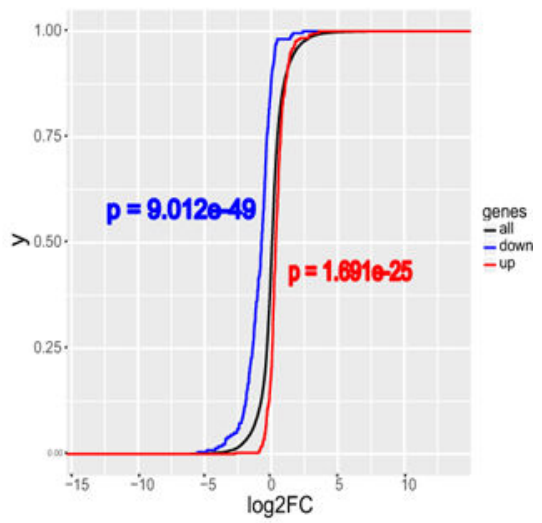

D

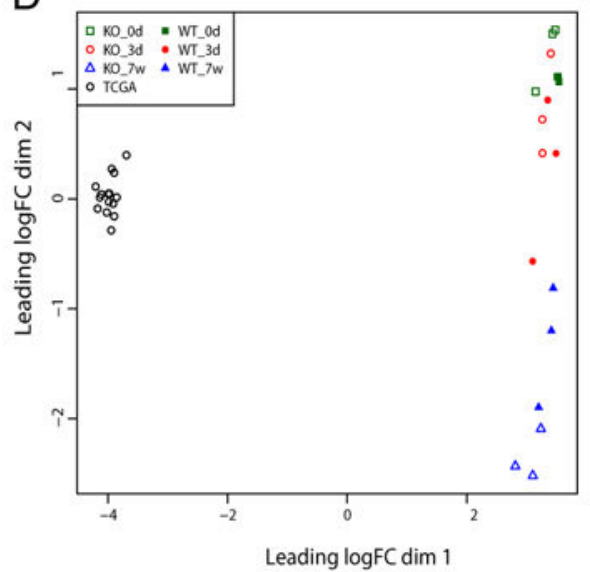

$\mathrm{E}$

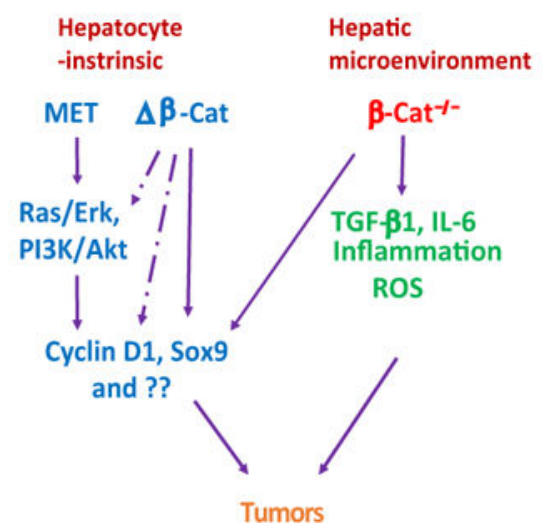

Figure 7. CAT/MET-induced tumor transcriptomes are shared in mice and humans

(A) Day 3 relative gene expression levels of all significant genes (all) were compared to genes up-regulated (up) or down-regulated (down) in tumors versus normal tissues. Onetailed Kolmogorov-Smirnov test.

(B) Week 7 relative gene expression levels of all significant genes (all) were compared to genes up-regulated (up) or down-regulated (down) in tumors versus normal tissues. Onetailed Kolmogorov-Smirnov test. 
(C) Correlation between week 7 mouse RNA-seq profiles with all TCGA-LIHC project tumor samples (TCGA_all) and samples with $C T N N B 1$ mutation and MET overexpression (TCGA_selected).

(D) MDS plot of RNA-seq profiles from TCGA-selected samples and mouse samples.

(E) A model for hepatocarcinogenesis synergistically enhanced by gained and lost functions of $\beta$-catenin in the liver. Exogenous expression of hMet and $\Delta$ N90- $\beta$-catenin drives proliferative signaling pathways and cell cycle progression. Meanwhile, deleting $\beta$-catenin in hepatocytes leads to dramatically enhanced production of pro-tumorigenic inflammatory effectors and ROS in the hepatic microenvironment, resulting in aggravated liver tumorigenesis. 